Research Article

\title{
Simulation and Optimization of Acoustic Package of Dash Panel Based on SEA
}

\author{
Jintao Su $\mathbb{D}^{1},{ }^{1}$ Ling Zheng $\mathbb{D}{ }^{1}{ }^{1}$ and Jianping Lou $^{2}$ \\ ${ }^{1}$ State Key Laboratory of Mechanical Transmission, Chongqing University, Chongqing 400044, China \\ ${ }^{2}$ Saic-iveco Hongyan Commercial Vehicle Co., Ltd., Chongqing 401122, China \\ Correspondence should be addressed to Ling Zheng; zling@cqu.edu.cn
}

Received 4 June 2020; Revised 5 September 2020; Accepted 1 October 2020; Published 29 October 2020

Academic Editor: Xin Xue

Copyright (c) 2020 Jintao Su et al. This is an open access article distributed under the Creative Commons Attribution License, which permits unrestricted use, distribution, and reproduction in any medium, provided the original work is properly cited.

The instrument panel assembly is an important structure to shield powertrain noise and front wheel noise, and the instrument panel sound insulation system plays a key role in it. Therefore, the sound insulation characteristics of the instrument panel sound insulation components appear particularly important. In the existing technology, the acoustic design of instrument panel mostly adopts the reverse design method and pays little attention to the forward design of acoustic system, which tends to lead to the shortcomings such as inaccurate acoustic design and poor acoustic design quality. The reverse design also restricts the development cycle, so the control and design of acoustic performance cannot be realized in the initial stage of design. Based on the above problems, the statistical energy model of the dashboard model was established by combining the statistical energy flow method and the proxy model method, and the influences of different acoustic cladding layers, different thickness, leakage, and new material microfibers on the sound insulation parameters of the front coaming were simulated. The general rules that affect the sound insulation performance of the structure are obtained. On this basis, multiobjective genetic algorithm and proxy model method are used to optimize the insertion loss of the front panel acoustic pack and the weight of the dashboard by introducing multiobjective variable and experimental design method, so as to obtain the best solution to meet the requirements of insertion loss and lightweight acoustic pack of the dashboard. It is of great engineering significance for the development of acoustic components for forward design instrument panel.

\section{Introduction}

With the rapid development of the automobile industry, the focus of consumers is not only on appearance, operation, fuel economy, and other aspects, but also on driver and passenger comfort. Important factors that lead to poor comfort include harmful car noise to the human body. Therefore, it is very important to improve comfort through effective sound insulation. The sound packaging of the instrument panel is a very important part in the sound insulation measures of the whole vehicle [1]. The front panel is an important part separating engine cabin and crew cabin. Its sound insulation performance determines to a great extent the interference degree of driver and passenger by engine noise. It is the premise of further analyzing, evaluating, and improving the sound insulation performance to accurately design the sound insulation performance of the system and components and to find out the main weak links by using reasonable and effective methods. It is of great significance to improve the acoustic performance of the automobile. At present, the existing sound insulation measurement methods can be divided into impedance tube method and laboratory method. Impedance tube method will be tested according to certain specifications in the impedance tube. Based on the microphone measuring sound pressure signal, meter laboratory method is to use the reverberation room and anechoic chamber. Tested on the whole thing, sound insulation quantity measurement method can consider the influence of the structure characteristics of the specimens, including reverberation chamber, reverberation chamber method, the reverberation room, and anechoic chamber method. Reverberation chamber and reverberation chamber method will use a reverberation 
chamber as sound room and another reverberation chamber as the reception room. The tested pieces are placed in between the reverberation chambers, based on the microphone in the two reverberation rooms, respectively, to measure average sound pressure signal sound insulation quantity calculation. This method needs to build two reverberation chambers and the facilities of repeated redundancy and increases the cost. The reverberation room-anechoic room method takes the reverberation room as the sounding room and the anechoic room as the receiving room. Compared with the reverberation room-anechoic room method, the anechoic room in this combination can be used not only for sound insulation measurement, but also for other acoustic tests such as noise source identification. Therefore, it is widely used in engineering. The acoustical bag of the dash panel is made up of porous and large damping materials such as foam and felt. It consumes medium and high frequency noise energy with the friction between materials and achieves the purpose of sound absorption and sound insulation [2]. Based on the SEA (Statistical Energy Analysis), aiming at the acoustic performance of panel optimization problem, power flow method based on statistical energy and $k$ Kriging agent model method, and the establishment of a sound insulation system dashboard statistical energy model and agent optimization model, we analyzed the area covered by different materials, sound insulation material thickness, and leakage holes and the influence of factors on the sound insulation performance dashboard. The present design method is improved; instead of adopting the reverse design method for acoustic arrangement, the new statistical energy method and proxy model method are adopted for the acoustic system design of the instrument panel, which has certain innovative significance. The instrument panel statistical energy model, reverberation chamber model, and anechoic chamber model were established to predict and verify the acoustic performance of instrument panel system, and the application of new materials in instrument panel design was proposed. In terms of the application of new materials, the thickness and surface density of new materials are taken as independent variables, and the sound insulation volume and the weight of acoustic package are taken as dependent variables, and the performance optimization design is carried out by using multiobjective genetic algorithm [3]. Finally, the optimal parameters of the instrument panel acoustic package are obtained. It has significance to the forward development and cost control of acoustic system of instrument panel system.

\section{SEA Theory}

The essence of SEA method is to divide the complex structural system or acoustic system into several coupled subsystems, analyzing and studying the energy transfer between subsystems under load $[4,5]$. A system is usually composed by many subsystems. By establishing the energyflow equation of the subsystem, the mass of the subsystem, the frequency range to be analyzed, the damping loss factor between the subsystems and the energy of the input system are determined. The average response energy of the subsystem in the corresponding frequency range is obtained by solving the energy flow equation, and it is converted into the required physical parameters. For the established statistical energy model, the following assumptions are proposed [6]:

(1) The coupling between subsystems is linear and conservative, and there is no nonconservative coupling.

(2) In the studied frequency range, energy is transferred between subsystems with resonance frequency.

(3) The excitation of the system is incoherent random excitation, independent of each other, with incoherence of modes, and the principle of linear addition of energy can be applied.

(4) In a given subsystem, the energy of all resonance modes in the given frequency band is equally divided.

(5) The reciprocity principle is applicable to different substructures.

(6) The energy flow between any two substructures is directly proportional to the actual energy difference between the coupled substructures during vibration; that is, the energy flow is directly proportional to the difference between the average modal energies. The energy model of the two subsystems is shown in Figure 1, and the energy flow relationship equation between the two subsystems is established:

$$
\begin{aligned}
& P_{1}=\omega \eta_{1} E_{1}+\omega \eta_{12} n_{1}\left(\frac{E_{1}}{n_{1}}-\frac{E_{2}}{n_{2}}\right), \\
& P_{2}=\omega \eta_{2} E_{2}+\omega \eta_{21} n_{2}\left(\frac{E_{2}}{n_{2}}-\frac{E_{1}}{n_{1}}\right),
\end{aligned}
$$

where $\eta=2 \xi$, it is generally composed by multiple systems, and these energy balance equations are written in matrix form:

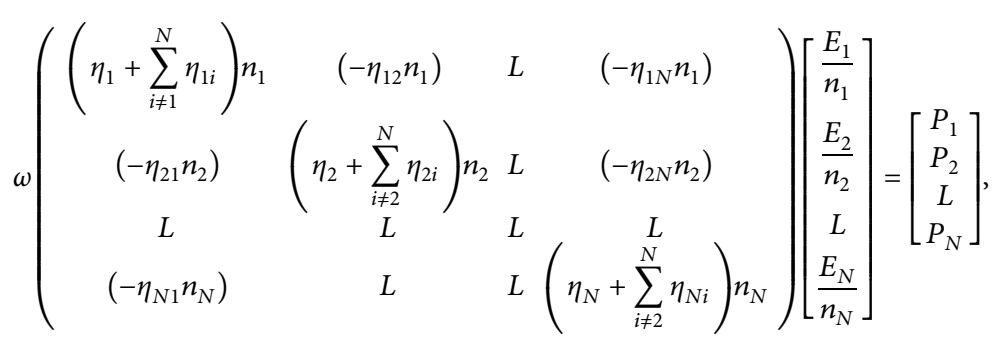




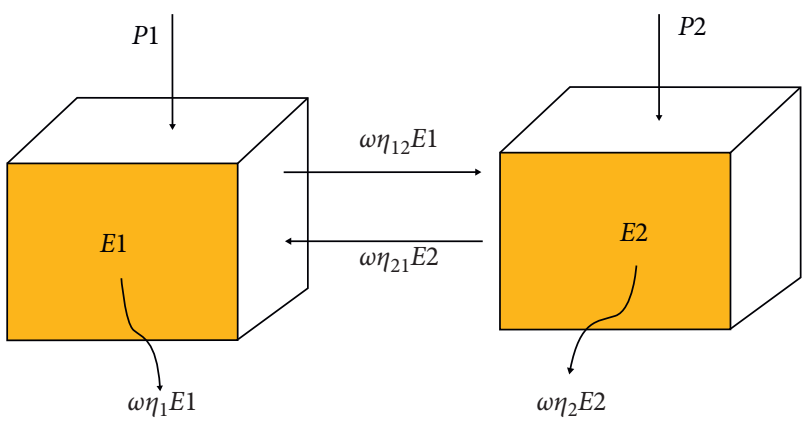

Figure 1: Energy analysis model of two subsystems.

where $\omega$ is the analysis center frequency $(\mathrm{Hz}), P_{i}$ is the input energy $(\mathrm{W}), \eta_{\mathrm{i}}$ is the damping loss factor, $\eta_{\mathrm{ij}}$ is the coupling damping loss factor, $E_{i}$ is the subsystem energy, and $\mathrm{Ni}$ is the subsystem modal density.

Equation (2) is the most basic expression of modeling by using SEA. When solving the calculation equation, under the condition of considering the external input excitation of the model, combined with the material test characteristics of the acoustic package structure, the loss factor, and modal density used between subsystems, the energy of the system is calculated; the energy $E_{i}$ on each subsystem is obtained [7]. According to (3), the energy system is converted into two forms, in which $E_{1}$ represents the energy of the structural subsystem. It depends on the mass and speed of the system. $E_{2}$ is the energy of the sound field. It is related to sound pressure, sound propagation speed, and other parameters in the sound field:

$$
E=\left\{\begin{array}{l}
E_{1}=M(V)^{2}, \\
E_{2}=\frac{V\left(P^{2}\right)}{\rho c^{2}},
\end{array}\right.
$$

where $M$ is the mass corresponding to each subsystem; $V$ is the vibration speed corresponding to the subsystem; $P$ is the sound pressure corresponding to the subsystem; $\rho$ is the material density of the subsystem; and $c$ is the sound speed corresponding to the material. By solving the equations, the energy contained in the corresponding system can be transformed into the corresponding displacement, subsystem speed, and strain, which can be measured.

\section{Simulation}

3.1. Building SEA Model. The finite element model of the dash panel is shown in Figure 2, and the thickness of the sheet metal is $0.8 \mathrm{~mm}$. The length and width of the front coaming are $1.6 \mathrm{~m} \times 0.6 \mathrm{~m}$. The front coaming is distributed in 9 holes with the thickness of $0.8 \mathrm{~mm}$ and the holes are of irregular shape. According to the SEA plate modeling method, nine SEA subsystems with irregular holes were established. Then acoustic cover is carried out according to different acoustic envelopment materials. In the division of subsystem, the structure with the same modal energy or modal damping is generally divided into a subsystem

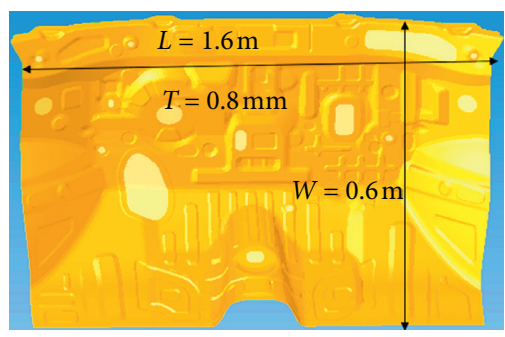

Figure 2: Finite element model of dash panel.

according to the principle of modal similarity of statistical energy method, or according to the geometric characteristics of the model. According to the above method, the dash panel is divided into 24 subsystems, and they are all plates structural subsystems. The $3 \mathrm{~mm}$ rubber simulation is used at the through-hole. According to the method in reference [8], the front coaming model is divided into subsystems. The partition principle is to divide the area with similar modal density into a subsystem and, at the same time, divide the area with complex structure and divide each hole. The division of subsystems is related to the energy of the system, so it is related to the calculation accuracy of the whole system. Each structural subsystem of SEA model is shown in Figure 3 and Table 1. The material parameters of SEA model of dash panel are shown in Table 2, and the acoustic cover material parameters of SEA model of dash panel are shown in Table 3.

3.2. Setting Boundary Condition. In order to simulate the reverberation room and semianechoic room, the sound cavity is set up on both sides of the dash panel. As shown in Figure $4,1 \mathrm{~Pa}$ unit sound pressure is loaded in the reverberation room. Figure 4(b) is the partial enlarged drawing of the dash panel; the creation of the reverberation room meets the national standard requirements GB/T20247 acoustic reverberation room sound absorption measurement. Solution frequency range is from $400 \mathrm{~Hz}$ to $8000 \mathrm{~Hz}$. The panel model is connected with two cavities, which simulate the semianechoic chamber and reverberation chamber, respectively. One cavity is loaded with sound excitation, and then the sound pressure levels of the two sound fields are tested, respectively. The front coaming model and two cavities are arranged in a semiwireless flow field.

The front coaming model of the mixed FE-SEA was placed between the reverberation chamber and the semianechoic chamber, as shown in Figure 4. In the figure, the left cavity simulates the semianechoic chamber and the right cavity simulates the reverberation chamber. The two sides of the sound cavity are connected by the front coaming model, and the left semianechoic chamber is stimulated by the sound source. Meanwhile, the front coaming model is set as a semi-infinite flow field, thus forming the front coaming hybrid FE-SEA sound absorption model composed of three subsystems. The mixing model satisfies FE-SEA mixing theory. Figure 4(b) is the front coaming model. The front coaming is composed of different substructures, including statistical energy plates and holes covered by acoustic cladding materials, forming a composite model of structure and porous materials. 


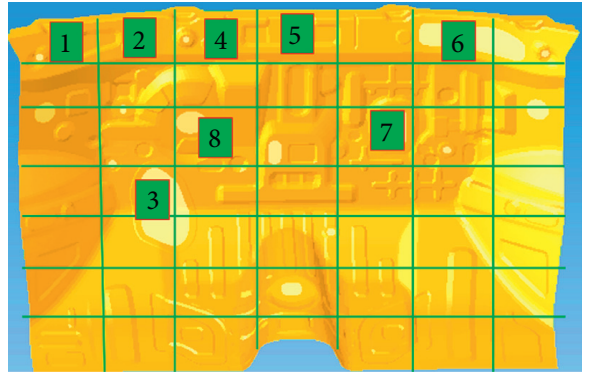

FIgURE 3: SEA model of dash panel.

TABle 1: Subsystem of dash panel.

\begin{tabular}{lccc}
\hline Number & Code name & Number & Code name \\
\hline 1 & Sheet metal 1 & 13 & Sheet metal 7 \\
2 & Sheet metal 2 & 14 & Sheet metal 8 \\
3 & Hole 1 & 15 & Sheet metal 9 \\
4 & Sheet metal 3 & 16 & Hole 7 \\
5 & Sheet metal 4 & 17 & Sheet metal 10 \\
6 & Hole 2 & 18 & Sheet metal 11 \\
7 & Hole 3 & 19 & Sheet metal 12 \\
8 & Hole 4 & 20 & Sheet metal 13 \\
9 & Hole 5 & 21 & Sheet metal 14 \\
10 & Hole 6 & 22 & Sheet metal 15 \\
11 & Sheet metal 5 & 23 & Sheet metal 16 \\
12 & Sheet metal 6 & 24 & Sheet metal 17 \\
\hline
\end{tabular}

\section{Experimental Method}

4.1. Parametric Test Method. The transmission loss test principle of the four-microphone method is shown in Figure 5. The sound source generates signals, and the sound pressure is amplified by the power amplifier. Sound pressure passes through the incident tube to generate the incident wave $P_{i}$, which is divided into three parts after passing through the sample. The first part is absorbed by the sample, and the second part is reflected by the sample, forming reflected wave $P_{r}$ in the incident tube. The third part continues to travel forward through the sample and forms the transmitted acoustic wave $P_{t}$ in the transmission tube. The transmitted wave continues forward to the acoustic terminal. One part is absorbed by the acoustic terminal and the other part is reflected into the transmission tube to form $p_{2 r}$. Four microphones are placed in the incident and transmission tubes to separate incident and reflected waves. The sound pressures measured at the position of sensors 1-4 are $p_{1}, p_{2}, p_{3}$, and $p_{4}$, respectively. In the incident tube, the incident sound pressure at the front end of the sample is PI. In the incident tube, the reflected sound pressure at the front end of the sample is $p_{r}$. In the transmission tube, the transmitted sound pressure is $p_{t}$. The sound pressure reflected through the sample is $p_{2 r}$. According to the propagation law of plane acoustic wave, the following formula can be obtained:

$$
\left\{\begin{array}{l}
p_{1}=p_{i} e^{j k\left(s_{1}+L_{1}\right)}+p_{r} e^{-j k\left(s_{1}+L_{1}\right)}, \\
p_{2}=p_{i} e^{j k L_{1}}+p_{r} e^{-j k L_{1}}, \\
p_{3}=p_{t} e^{j k L_{2}}+p_{2 r} e^{-j k L_{2}}, \\
p_{4}=p_{t} e^{j k\left(s_{2}+L_{2}\right)}+p_{2 r} e^{-j k\left(s_{2}+L_{2}\right)},
\end{array}\right.
$$

where $k$ is the wavenumber, which can be obtained from the following equation:

$$
k=\frac{2 \pi f}{c}
$$

where $f$ is the frequency, $c$ is the speed of sound-generally, it is $340 \mathrm{~m} / \mathrm{s}$, and $k$ is the wavenumber. The above formula can be used to calculate the incident sound pressure $p_{\mathrm{I}}$, reflected sound pressure $p_{r}$, transmitted sound pressure $p_{t}$, and reflected sound pressure $p_{2 r}$ at the acoustic end.

$$
\left\{\begin{array}{l}
p_{i}=\frac{p_{1}-p_{2} e^{-j k s_{1}}}{e^{j k s_{1}}-e^{-j k s_{1}}} e^{-j k L_{1}}, \\
p_{r}=\frac{p_{1}-p_{2} e^{-j k s_{1}}}{e^{-j k s_{1}}-e^{j k s_{1}}} e^{j k L_{1}}, \\
p_{t}=\frac{p_{3} e^{j k s_{2}}-p_{4}}{e^{j k s_{2}}-e^{-j k s_{2}} e^{j k L_{2}}}, \\
p_{2 r}=\frac{p_{3} e^{-j k s_{2}}-p_{4}}{e^{-j k s_{2}}-e^{j k s_{2}} e^{-j k L_{2}}} .
\end{array}\right.
$$

Therefore, the sound pressure transmission coefficient of the sample is

$$
t_{p}=\frac{p_{t}}{p_{i}}=\frac{\sin \left(k s_{1}\right)}{\sin \left(k s_{2}\right)} \frac{p_{3} e^{j k s_{2}}-p_{4}}{p_{1}-p_{2} e^{-j k s_{1}}} e^{j k\left(L_{1}+L_{2}\right)} .
$$

Transmission loss of the sample:

$$
\mathrm{TL}=-201 g\left|t_{p}\right| \text {. }
$$

Compared with the three-microphone method, the four-microphone method uses two microphones in the transmission tube, and the measurement results are more accurate. It can measure the reflection coefficient of sample, sound source, and acoustic terminal. In the sound insulation test, the front coaming material is cut into a circular plate and the acoustic absorbing materials are put into the impedance tube for sound insulation test. The size of the front coaming sample and the acoustic absorbing material sample is a circle cake with a radius of $50 \mathrm{~mm}$.

Flow resistance is the resistance of air through gaps in the material, per unit thickness. The flow resistance is called the flow resistance rate of the material. The flow resistance rate is tested by controlling one-way flow. The air flow passes through the porous material in the cylindrical tube and the porous material table is measured. Pressure difference is generated by surface. This can be affected if there is an air gap between the material and the container. The accuracy of the results on the foamed material is due to the influence of the processing technology. A sticky film on the surface may also affect the accuracy of the measurement results. In this paper, use $100 \mathrm{~mm}$ diameter sample with ring, add one ring, and then add two. The flow resistance rate of foam was tested under three boundary conditions (see Figure 6). The test standard complies with ISO 9053-91 and ASTMC5522-03 standards. Figure 6 is the schematic diagram of flow 
TABLE 2: Materials parameter of SEA model.

\begin{tabular}{lcccc}
\hline Material & Density $\left(\mathrm{kg} / \mathrm{m}^{3}\right)$ & Tensile modulus $(\mathrm{Pa})$ & Shear modulus $(\mathrm{Pa})$ & Poisson's ratio \\
\hline Steel & 7800 & $2.1 \mathrm{e}+11$ & $8 \mathrm{e}+10$ & 0.3125 \\
Rubber & 1100 & $2.3 \mathrm{e}+9$ & $7.72 \mathrm{e}+8$ & 0.4896 \\
\hline
\end{tabular}

TABle 3: Acoustic cover material parameters.

\begin{tabular}{lccccccc}
\hline Material & Density $\left(\mathrm{kg} / \mathrm{m}^{3}\right)$ & Tensile modulus $(\mathrm{Pa})$ & Poisson's ratio & Loss coefficient & Porosity & Curvature & Fluid resistivity $\left(\mathrm{N} . \mathrm{s} / \mathrm{m}^{4}\right)$ \\
\hline Cast foam & 22 & $6.5 \mathrm{e}+4$ & 0.4 & 0.15 & 0.94 & 1.3 & $2.6 e+4$ \\
Felt & 50 & - & - & 0.4 & - & 0.92 & 1.5 \\
PU foam & 22 & $2.65 \mathrm{e}+4$ & 0.4 & - & 0.96 & 1.24 & $4.5 e+4$ \\
EVA & 1670 & $2.1 \mathrm{e}+9$ & - & - & 5000 \\
\hline
\end{tabular}

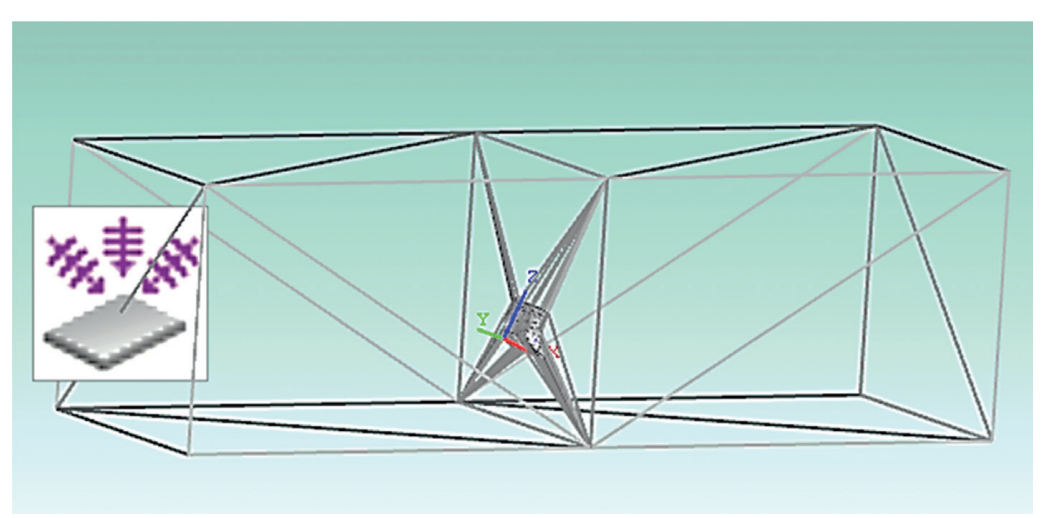

(a)

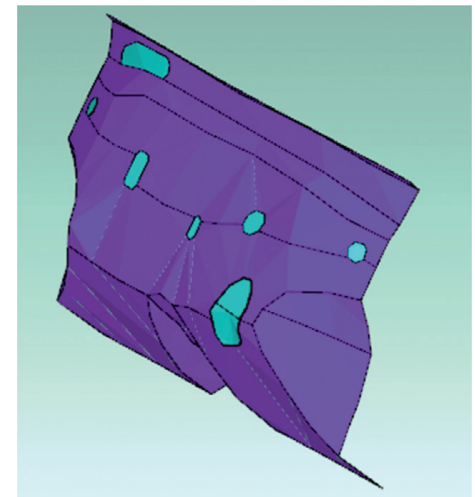

(b)

Figure 4: Boundary condition.

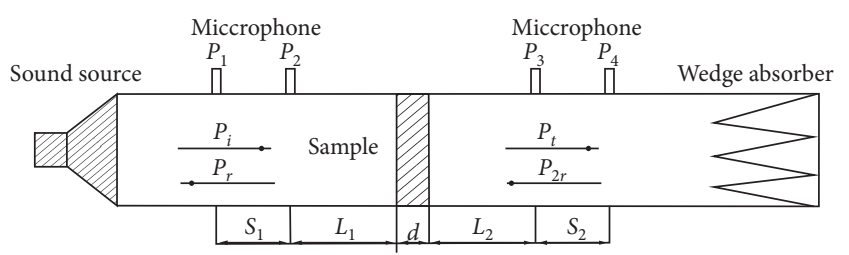

FIGURE 5: Transmission loss test principle of the four-microphone method.

resistance rate test, and Figure 6(a) is the flow resistance rate test equipment. The flow resistance rate of the porous material sample is tested through the pressure difference at both ends of the test sample and the volume velocity per unit area. Figure 6(b) shows the sample fabrication and postprocessing of the experimental data for the flow resistance rate test. The acoustic properties of porous materials can be obtained by measuring the flow resistance rate of porous materials.

\section{Model Validation}

5.1. Model Verification Based on Sound Insulation. The verification of the simulation model of the front coaming with different thickness is shown in Figures 7 and 8 . It can be seen from Figure 7 that the error between the simulation result and the test result is small, and the simulation value is in good agreement with the test value after the refined mesh correction of the front coaming model. It can be seen from Figure 7 that the simulation value of transfer loss after model modification is consistent with the test value in the whole frequency range. When the frequency is less than $600 \mathrm{~Hz}$, there is a peak error in the modified model sound transfer loss, which is mainly caused by the sound radiation of the plates. With the increase of frequency, the error decreases gradually. When the frequency range is $600-2000 \mathrm{~Hz}$, the error of simulation results and test results after model modification is less than $3 \mathrm{~dB}$. The modified model can be used for performance calculation and performance prediction, and the model is accurate and reliable.

By the same token, the thickness of $0.8 \mathrm{~mm}$ is presented in Figure 8, which shows the dash panel of the results of simulation and test results contrast. From the diagram, it is shown that, in low frequency range of $0-800 \mathrm{~Hz}$, the simulation value and test value error is small, which is almost the same curve trend. With the improvement of frequency, error increases, as the model of fine grid modeling and model, the simulation value and test and the simulation model accuracy are reliable and we can carry out models prediction. Similarly, as shown in Figure 8, the error between the simulation value and the test value of the model decreases gradually after modification, which can be used for simulation and performance prediction of the model. 


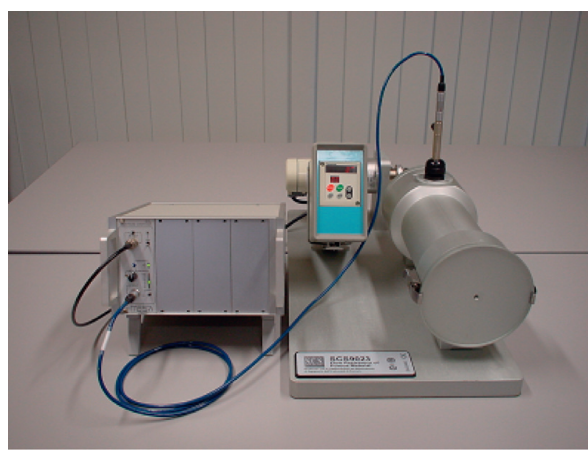

(a)

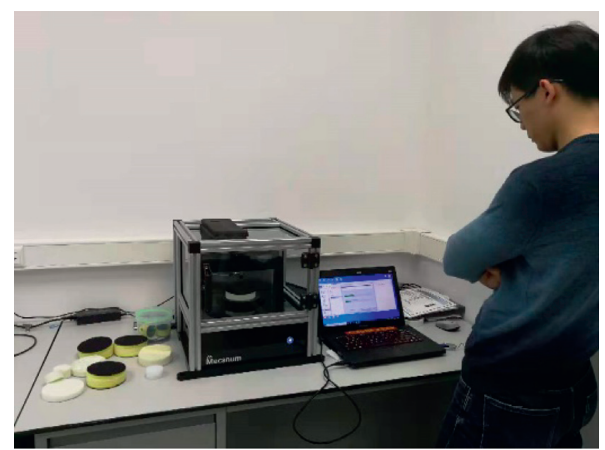

(b)

Figure 6: Flow resistance test picture.

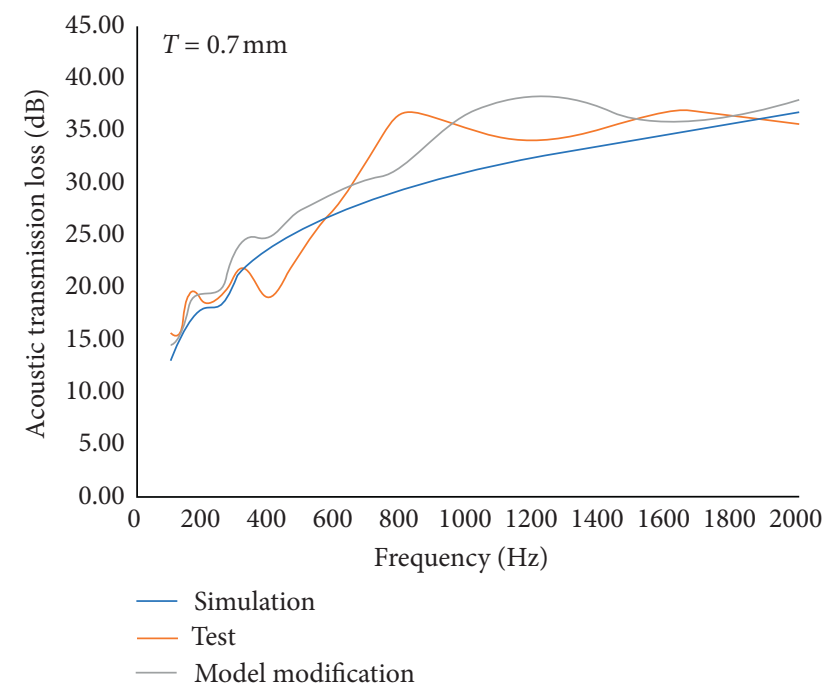

FIGURE 7: Validation of the front coaming model with a thickness of $0.7 \mathrm{~mm}$.

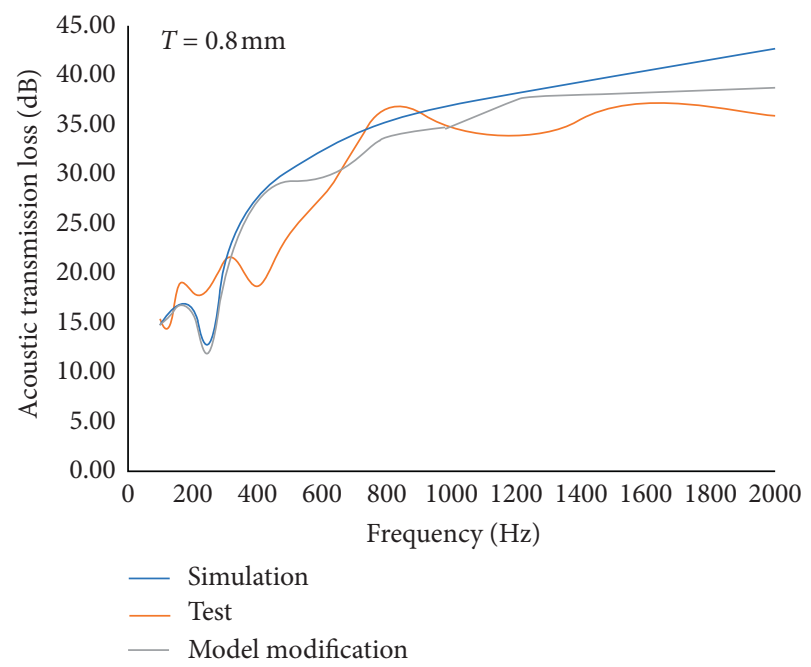

FIgURE 8: Validation of the front coaming model with a thickness of $0.8 \mathrm{~mm}$.

\section{Transmission Loss Effect}

6.1. Impact Analysis of Sound Insulation Performance. Sound insulation performance is usually described by sound insulation $(R)$, noise attenuation (NR), and insertion loss (IL). The sound insulation performance of the dash panel's interior trim is evaluated by insertion loss, that is, the difference value between the incident sound intensity level $\left(L_{0}\right)$ and the transmitted sound intensity level $(L)$ [9]:

$$
\mathrm{IL}=L_{0}-L(d B) \text {. }
$$

6.1.1. Impact of Coverage. In order to analyze the impact of interior trim under different covering conditions on the sound insulation performance of the dash panel, four kinds of acoustic package covering schemes are given, as shown in Table 4.

(1) Base scheme: the inside is without acoustic package.

(2) Scheme 1: the inside of the dash panel is covered by $25 \mathrm{~mm}$ acoustic package ( $23 \mathrm{~mm} \mathrm{PU}+2 \mathrm{~mm}$ EVA), the outside has no coverage, and the hole is covered by $25 \mathrm{~mm}$ acoustic package $(10 \mathrm{~mm}$ cast foam $+15 \mathrm{~mm}$ felt).

(3) Scheme 2: the inside of the front dash is covered by $25 \mathrm{~mm}$ acoustic package ( $23 \mathrm{~mm} \mathrm{PU}+2 \mathrm{~mm}$ EVA), the outside has no coverage, and the hole is covered by $10 \mathrm{~mm}$ cast foam.

(4) Scheme 3: the inside of the front dash is covered by $25 \mathrm{~mm}$ acoustic package ( $23 \mathrm{~mm} \mathrm{PU}+2 \mathrm{~mm}$ EVA), and the outside and the hole have no coverage.

The comparison curve of sound insulation performance of four kinds of acoustic package coverage is shown in Figure 9. Comparing Scheme 3 with base scheme, the acoustic materials help to improve its sound insulation performance. Referring to the curve in the figure, the difference of insertion loss between the two schemes is the largest at the frequency $2000 \mathrm{~Hz}$, which is $11.4 \mathrm{~dB}$. Comparing Scheme 1 with Scheme 3, after hole coverage is increased, the sound insulation performance is improved with the increase of frequency, and the insertion loss difference between the two schemes meets the maximum at $8000 \mathrm{~Hz}$, 
TABLE 4: Cover means of acoustic package.

Without acoustic package Scheme 1

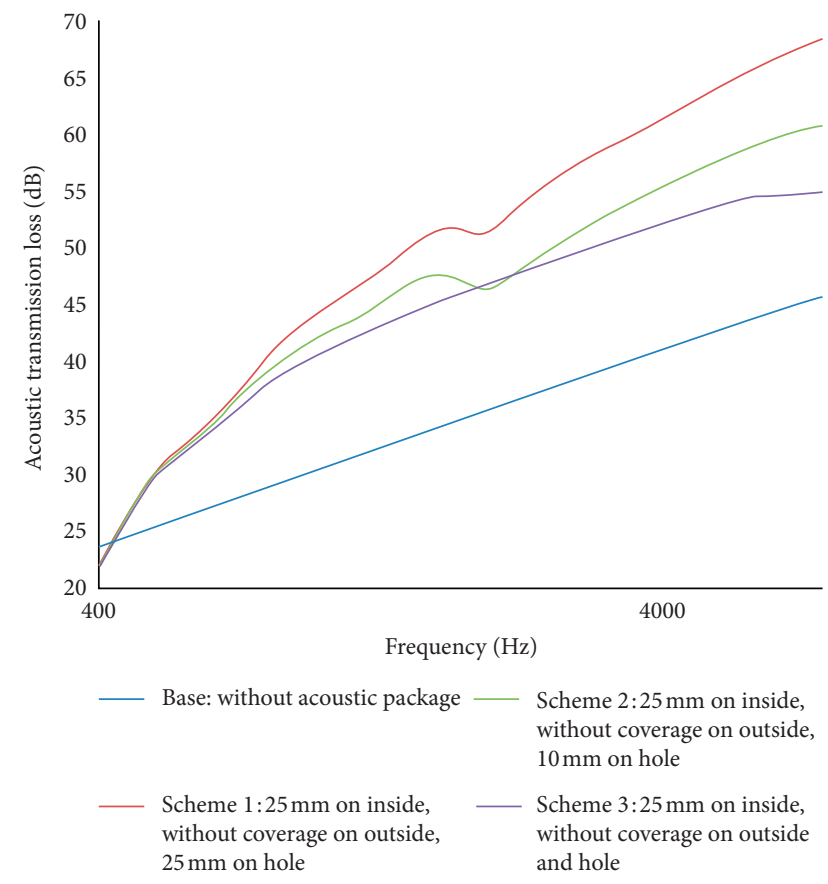

FIGURE 9: Impact of coverage of sound insulation performance.

which is $13 \mathrm{~dB}$. It can be seen that the acoustic material coverage of the sheet metal and holes of the cowl dash panel has a great influence on the sound insulation performance. Therefore, when the acoustic cladding material of the same thickness is covered, the hole of the panel is the key factor affecting the sound transmission loss, and the sound transmission loss increases with the increase of the thickness of the hole coverage area, and the hole leakage is in direct proportion to the sound transmission loss. In the design of acoustic envelopment system, the acoustic envelopment covering and holes are the key points of the design.

6.1.2. Impact of Thickness. In order to analyze the impact of the thickness of acoustic package material and through-hole plug on the sound insulation performance of the dash panel, three schemes are designed, as shown in Table 5.
(1) Schemes 1: the inside of the dash panel is covered by $25 \mathrm{~mm}$ acoustic package ( $23 \mathrm{~mm} \mathrm{PU}+2 \mathrm{~mm}$ EVA), without coverage on the outside and holes.

(2) Schemes 2: the inside of the dash panel is covered by $25 \mathrm{~mm}$ acoustic package ( $23 \mathrm{~mm} \mathrm{PU}+2 \mathrm{~mm}$ EVA), the outside has no coverage, and the hole is covered by $10 \mathrm{~mm}$ cast foam.

(3) Schemes 3: the inside of the cowl panel is covered by $15 \mathrm{~mm}$ acoustic package (13 $\mathrm{mm} \mathrm{PU}+2 \mathrm{~mm}$ EVA), while the outer side and the hole are not covered.

Figure 10 shows the comparison curve of sound insulation performance of three kinds of acoustic package material's thickness. It can be seen from the figure, compared with Scheme 2, that Scheme 1 has a certain attenuation of sound insulation performance with the increase of frequency, and the attenuation reaches $5 \mathrm{~dB}$ at $8000 \mathrm{~Hz}$. It can be seen that the thickness of the through-hole plug has a greater impact on the sound insulation performance. Compared with Scheme 3, the sound insulation performance of Scheme 1 is enhanced when the frequency is from $400 \mathrm{~Hz}$ to $2000 \mathrm{~Hz}$, but after $2000 \mathrm{~Hz}$; the corresponding curves of the two schemes are coincident, and there is no difference between the two schemes. It can be concluded that, at low frequency, the increase of material thickness has an enhanced impact on sound insulation. On the contrary, at high frequency, it has no effect. Therefore, in the design of acoustic envelopment scheme, increasing the thickness of acoustic envelopment material can effectively increase the sound transmission loss within the frequency range of $0-2000 \mathrm{~Hz}$. When the frequency is greater than $2000 \mathrm{~Hz}$, increasing the material thickness has little influence on the sound transmission loss, while the sound leakage quantity has great influence on the sound transmission loss. With the same material thickness, the maximum transmission loss in the high frequency range can be reduced by $5 \mathrm{~dB}$ after the sound leakage is blocked.

6.1.3. Impact of Leakage. When analyzing the impact of leakage on the sound insulation performance of dash panel, three schemes are proposed, as shown in Table 6. 
TABle 5: Coverage thickness of three schemes.

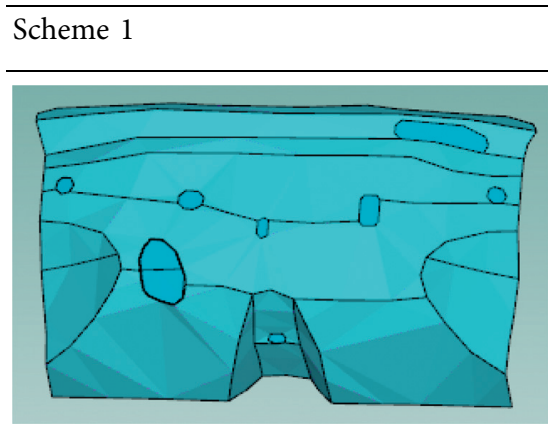

$25 \mathrm{~mm}$ on inside, without coverage on outside and hole

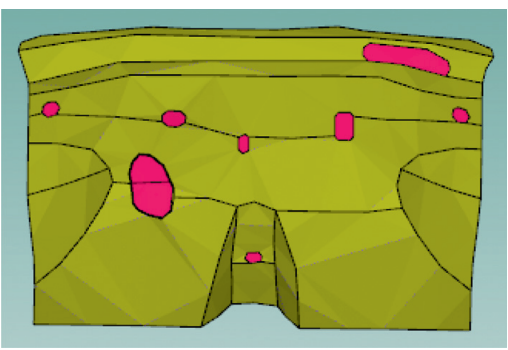

$25 \mathrm{~mm}$ on inside, without coverage on outside, $10 \mathrm{~mm}$ on hole
Scheme 3

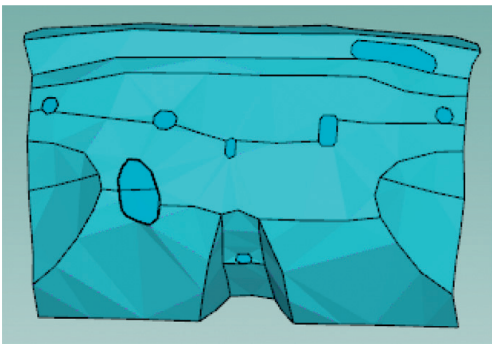

$15 \mathrm{~mm}$ on inside, without coverage on outside and hole

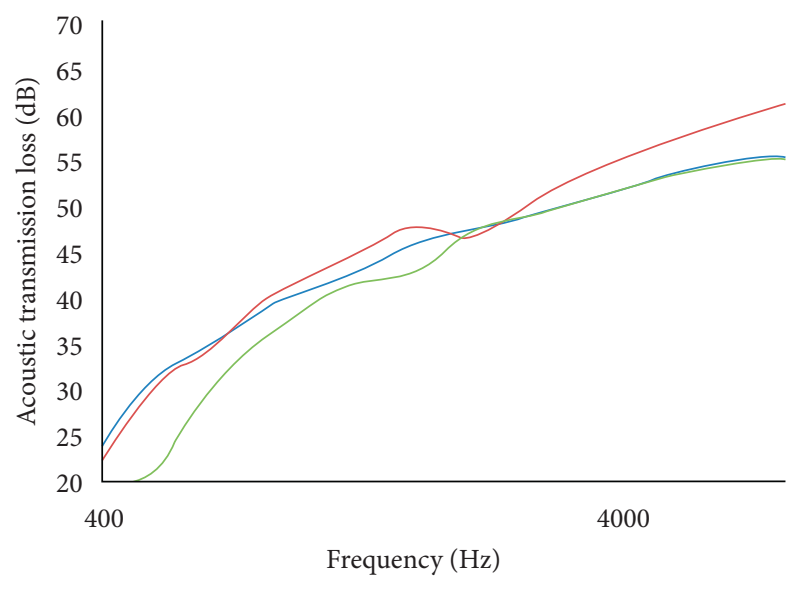

- Scheme 1: $25 \mathrm{~mm}$ on inside, without coverage on outside, and hole

- Scheme 2: $25 \mathrm{~mm}$ on inside, without coverage on outside, $10 \mathrm{~mm}$ on hole

- Scheme 3: $15 \mathrm{~mm}$ on inside, without coverage on outside, on hole

FIGURE 10: Impact of thickness on sound insulation performance.

TABLE 6: Three schemes of leakage.

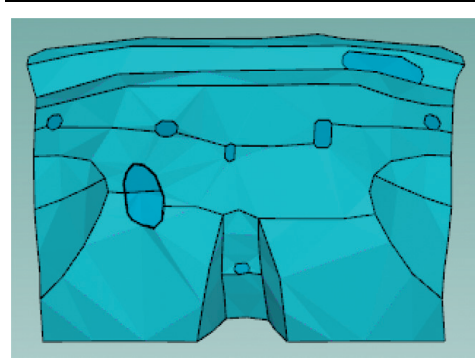

$27 \mathrm{~mm}$ on inside

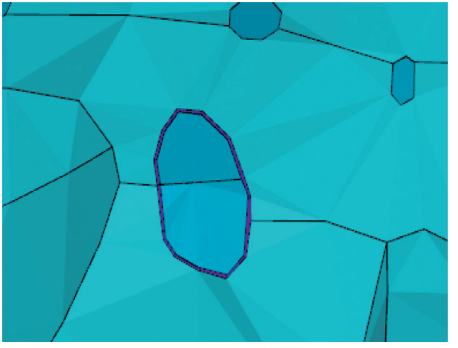

Steering string exposed $3 \mathrm{~mm}$

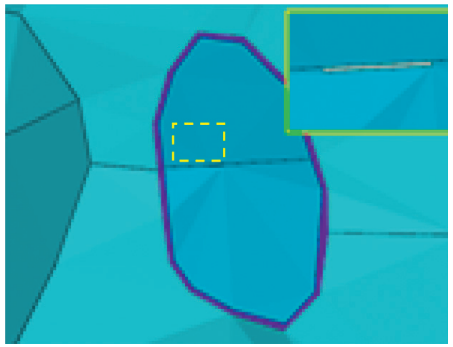

Steering string leakage area $1 \mathrm{~cm} * 0.5 \mathrm{~mm}$ 
(1) Scheme 1: the inside of the dash panel is covered by $27 \mathrm{~mm}(25 \mathrm{~mm} \mathrm{PU}+2 \mathrm{~mm} \mathrm{EVA})$, and the hole and the outside are not covered.

(2) Scheme 2: on the basis of Scheme 1, the exposed sheet metal of steering is considered, and the width is $3 \mathrm{~mm}$.

(3) Scheme 3: based on Scheme 2, the possible leakage of the steering is considered, and the area is $1 \mathrm{~cm}^{*}$ $0.5 \mathrm{~mm}$.

Figure 11 shows the compared curve of sound insulation performance of three schemes. It can be seen from the figure that when there is leakage on the dash panel (Scheme 3), compared with Scheme 1, its sound insulation performance has a certain reduction, reaching $2.5 \mathrm{~dB}$ at $8000 \mathrm{~Hz}$. So the leakage should be strictly controlled in the design of dash panel's interior trim.

\section{Application of New Materials in the Dash Panel of Automobile}

7.1. Introduction of New Materials. There are several kinds of commonly used materials for acoustic package of dash panel [10], such as PU foam layer + EVA double layer, felt + EVA double layer, and double impedance felt. The front two kinds of EVA sound insulation pads perform well in sound insulation performance, but the weight of the sound insulation pad is large, which hinders the lightweight of the dash panel of the car. Double impedance felt can achieve a good balance in sound absorption and insulation, and its quality is lighter than the front two materials. However, the VOC content of the above three forms of acoustic pad is high; it does not cause environmental protection and is not beautiful.

Microfiber material $[11,12]$ is a new material with a structure of 2-micron microfiber. The average diameter of the fiber is about $1 / 40$ of human hair. Under the same acoustic performance conditions, only fewer materials are needed. Under the same areal density, the acoustic performance is better. It also can better meet the needs of lightweight design and achieve the goal of reducing fuel consumption and carbon emissions. In addition to good acoustic performance, lightweight, high formability, and beautiful appearance, microfiber material also has the advantages of ultralow volatility and hydrophobic and mildew proof.

7.2. Simulation Results of Sound Insulation Performance of New Materials. In order to analysis the absorption and sound insulation capacity of the new materials, traditional acoustic materials and formable microfiber materials are covered on the inside of the car's dash panel. The specific scheme is shown in Table 7.

Scheme 1: the inside of the dash panel is covered by $27 \mathrm{~mm}$ traditional material ( $25 \mathrm{~mm} \mathrm{PU}+2 \mathrm{~mm}$ EVA), the outside of the dash panel is covered by $25 \mathrm{~mm}$ $(2.5 \mathrm{~mm}$ nonwoven fabric $+20 \mathrm{~mm} \quad \mathrm{PU}+2.5 \mathrm{~mm}$

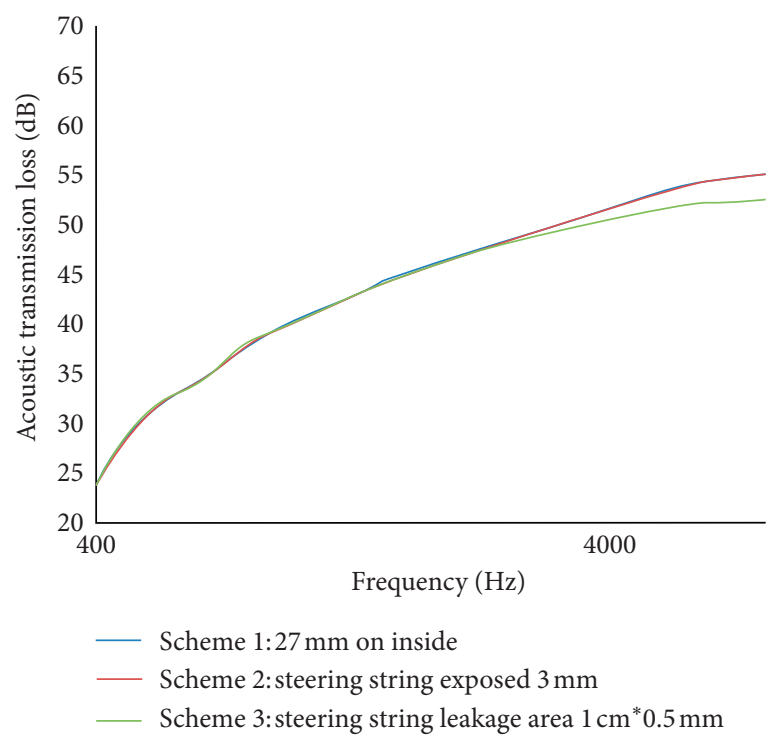

FIGURE 11: Compared curve of sound insulation performance of three schemes.

nonwoven fabric), and the hole is not covered with any acoustic material.

Scheme 2: the inside of the cowl panel is covered by $27 \mathrm{~mm}$ new material, the outer side of the cowl panel is covered with $25 \mathrm{~mm} \quad(2.5 \mathrm{~mm}$ nonwoven fabric $+20 \mathrm{~mm} \mathrm{PU}+2.5 \mathrm{~mm}$ nonwoven fabric), and the hole soundless material is covered.

Figure 12 shows the compared curve of sound insulation performance between the two schemes. It can be seen from the simulation results in the figure that the sound insulation effect of the whole frequency band is basically the same as that of the traditional PU +EVA material when the new material is covered on the inside of the dash panel, which shows that the new material can meet the sound insulation performance requirements. At the same time, under the same sound insulation performance, the surface density is $1375 \mathrm{~g} / \mathrm{m}^{2}$. It is also far lower than that of PU + EVA (4840 g/ $\mathrm{m}^{2}$ ). Therefore, dash panel's sound insulation pad with microfiber material can better meet the needs of lightweight design and achieve the goal of reducing fuel consumption and carbon emissions.

\section{Multiobjective Optimization}

8.1. Experimental Design. The initial sample points need to be obtained by computer simulation experiments. Different design methods have different coverage of sample points, and their distribution has a great impact on the accuracy of the agent model. Latin hypercube sampling (LHS) [13] is one of the most widely used sampling methods nowadays. The key of this method is to stratify the input probability distribution, divide the cumulative curve $(0 \sim 1)$ into equal intervals on the cumulative distribution function, and randomly select samples from each interval to forcibly represent the interval. Figure 13 is a typical LHS. 
Table 7: Two kinds of covered schemes.

Scheme 1

Scheme 2

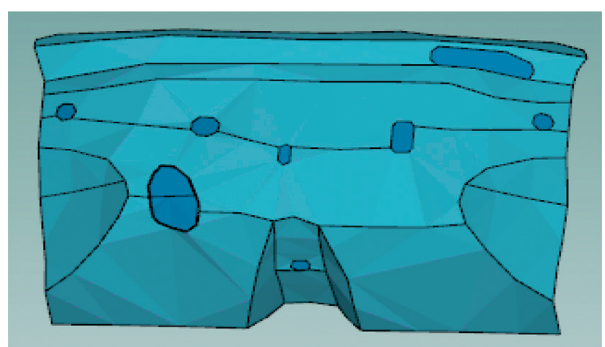

Inside covered by $27 \mathrm{~mm}$ (traditional material) Outside covered by $25 \mathrm{~mm}$, hole has no coverage

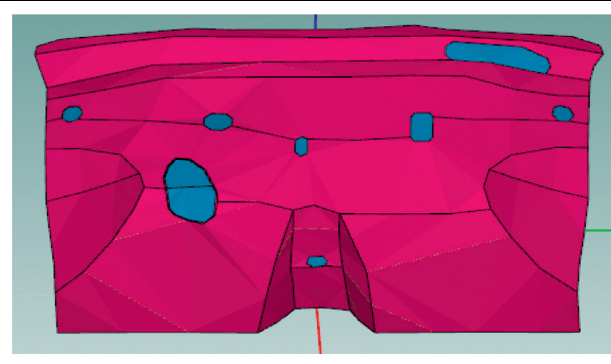

Inside covered by $27 \mathrm{~mm}$ (new material) Outside covered by $25 \mathrm{~mm}$, hole has no coverage

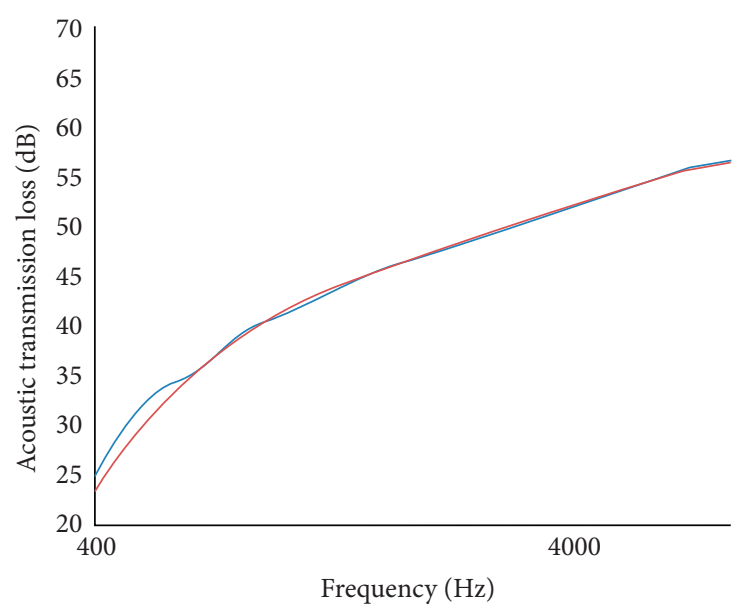

— Scheme 1:inside covered by

$27 \mathrm{~mm}$ (traditional meterial) outside covered by $25 \mathrm{~mm}$, hole has no coverage

- Scheme 2:inside covered by $27 \mathrm{~mm}$ (new meterial) outside covered by $25 \mathrm{~mm}$, hole has no coverage

FIGURE 12: Compared curve of sound insulation performance.

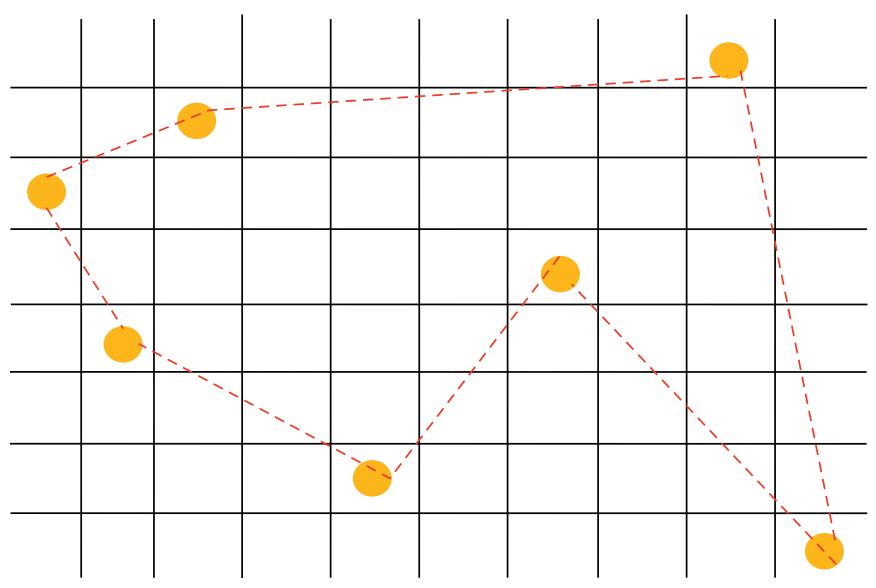

FIGURE 13: Latin hypercube sampling. 
In this paper, the new material of acoustic bag of automobile is taken as the research object, the thickness and area density of the material are taken as the design variables, and the average insertion loss and material weight are taken as the optimization objectives. The greater thickness and surface density of sound absorption and insulation materials generally have better sound absorption and insulation performance. However, due to the limitation of the actual vehicle space and the lightweight demand of the whole vehicles, the material can not be used without limit. We set the thickness range of the sound absorption part of the new material as $10 \sim 25 \mathrm{~mm}$ in this paper and the surface density range of the sound insulation part (at surface forming part) as $1000 \sim 1500 \mathrm{~g} / \mathrm{m}^{2}$. The LHS method is used to randomly select 20 groups of samples. Each group of samples can use SEA model to calculate the average insertion loss in the frequency band of $400 \sim 8000 \mathrm{~Hz}$. It is shown in Table 8 .

8.2. Kriging Agent Model. The agent model, also known as meta model or response surface, describes the functional relationship between output variables and input variables. In order to reduce the time of numerical analysis in the optimization process, the agent model is proposed, which uses limited sample points to construct an approximate simplified model instead of the complex practical analysis process. Therefore, it is widely used in multiobjective design optimization calculation. Kriging $[14,15]$ model is a semiparametric interpolation method. Its regression equation consists of two parts: parametric process (linear regression part) and nonparametric process (random process).

In this paper, with the information of 20 groups of sample points mentioned above, with the help of MATLAB software, Kriging model is used to predict the average insertion loss and weight of new materials in the design space. The results are shown in Figure 14.

8.3. The Verification of Model's Accuracy. In the generalized linear regression analysis, the goodness of fit $\left(R^{2}\right)$ is usually used to measure the goodness of fit of the model. The range of $R^{2}$ is $[0,1]$; the closer the value is to 1 , the higher the precision of the agent model is. The following equation is the expression of $R^{2}$ :

$$
R^{2}=1-\frac{\sum_{i=1}^{p}\left(y_{i}-\widehat{y}_{i}\right)^{2}}{\sum_{i=1}^{p}\left(y_{i}-\bar{y}_{i}\right)^{2}},
$$

where $P$ is the number of sample points and $y_{i}, \hat{y}_{i}$, and $\bar{y}_{i}$ are true value, predicted value, and average value of the true value of the response. In engineering, $R^{2}$ value is required to be above 0.9 . In order to verify the accuracy of the constructed agent model, we use Latin hypercube sampling (LHS) to randomly select another 10 groups of samples. Similarly, we can use sea model to calculate the average insertion loss and total material weight of the test samples. At the same time, the statistical energy method is used to calculate and compare the measured values to obtain the real values. According to (10), the goodness of fit was calculated. According to the comparison between the calculated results and the insertion loss obtained by the agent model, the good fitting degree of the agent model is obtained and the specific information is shown in Table 9. From the calculation results of goodness of fit, it can be seen that the goodness of fit of the agent model is above 0.9 and close to 1 . The proxy model has high precision, so the prediction result of approximate model is reliable.

8.4. Multiobjective Optimization of Dash Panel. The optimization of acoustic packaging for automobile dash panel belongs to a multiobjective optimization problem. The design variables generally are thickness and surface density of new acoustic materials for dash panel, and the optimization objectives are insertion loss and acoustic package's weight. These objectives not only are related to each other but also conflict with each other. To improve one of these objectives, it is often at the expense of other objectives. In this paper, the toolbox of multiobjective genetic algorithm [16] built in MATLAB is used to optimization. After 200 steps of iteration, Pareto solution set between average insertion loss and total weight of material is obtained. It is shown in Figure 15. As shown in Figure 14, the pentagram represents a series of Pareto optimal solutions, which represents the relationship between average insertion loss and material weight under different weight considerations. According to the lightweight requirements of the acoustic materials of dash panel, we need to control the weight of the new below $2 \mathrm{~kg}$. At the same time, the optimal values of thickness and surface density are $24.87773 \mathrm{~mm}$ and $1363.959 \mathrm{~g} /$ $\mathrm{m}^{2}$. The corresponding average insertion loss is $44.5629 \mathrm{~dB}$, and the total weight is $1.9958 \mathrm{~kg}$. In this paper, the optimal solution of acoustic envelopment is obtained according to the agent model and the mixed SEA method. Under the condition of satisfying the design of sound insulation quantity, the Latin hypercube theory of agent model is applied to design the optimal solution of acoustic envelopment material thickness and surface density, which meets the needs of engineering design.

Input the optimal values of the above thickness and surface density into SEA model, calculating the average insertion and total weight of acoustic materials under the value by software, and comparing the results with those results obtained by Kriging model. It is shown in Table 10.

According to the data in Table 10, the deviations of average insertion loss and material weight between the two calculated models, respectively, are $0.2 \%$ and $0.065 \%$. It indicates that the fitting accuracy of this approximate model is high. Therefore, it has a certain practical value to optimize the parameters of acoustic package materials by multiobjective genetic algorithm. It is concluded from the calculation time of the agent optimization model that the calculation time of the agent optimization model is much less than that of the statistical energy model for the complex acoustic treatment model of the front cob. The calculation accuracy and efficiency are further improved, which has certain reference significance for the optimization of engineering problems.

\section{Summary}

(1) In this paper, the forward design method of acoustic packet theory is studied. Statistical energy method 
TABLE 8: 20 groups of samples of LHS.

\begin{tabular}{|c|c|c|c|c|}
\hline Sample & Surface density of compress fiber $\left(\mathrm{g} / \mathrm{m}^{2}\right)$ & Thickness of glass fiber (mm) & Average insertion loss $(\mathrm{dB})$ & Total weight $(\mathrm{kg})$ \\
\hline 1 & 1275.482 & 24.774 & 44.293 & 1.876 \\
\hline 2 & 1442.005 & 13.279 & 42.838 & 2.013 \\
\hline 3 & 1009.287 & 21.359 & 43.437 & 1.497 \\
\hline 4 & 1033.835 & 14.589 & 42.519 & 1.481 \\
\hline 5 & 1054.355 & 23.030 & 43.725 & 1.570 \\
\hline 6 & 1230.138 & 10.503 & 42.055 & 1.712 \\
\hline 7 & 1341.836 & 16.172 & 43.203 & 1.902 \\
\hline 8 & 1490.006 & 18.521 & 43.770 & 2.116 \\
\hline 9 & 1471.053 & 17.541 & 43.589 & 2.083 \\
\hline 10 & 1100.610 & 19.866 & 43.395 & 1.608 \\
\hline 11 & 1373.022 & 12.581 & 42.625 & 1.917 \\
\hline 12 & 1376.294 & 20.903 & 43.964 & 1.982 \\
\hline 13 & 1409.591 & 16.763 & 43.384 & 1.996 \\
\hline 14 & 1077.093 & 13.853 & 42.461 & 1.533 \\
\hline 15 & 1261.767 & 22.387 & 43.990 & 1.841 \\
\hline 16 & 1147.143 & 19.672 & 43.443 & 1.668 \\
\hline 17 & 1303.536 & 24.029 & 44.251 & 1.908 \\
\hline 18 & 1155.843 & 11.044 & 42.068 & 1.617 \\
\hline 19 & 1180.924 & 15.862 & 42.941 & 1.685 \\
\hline 20 & 1220.306 & 12.237 & 42.369 & 1.711 \\
\hline
\end{tabular}

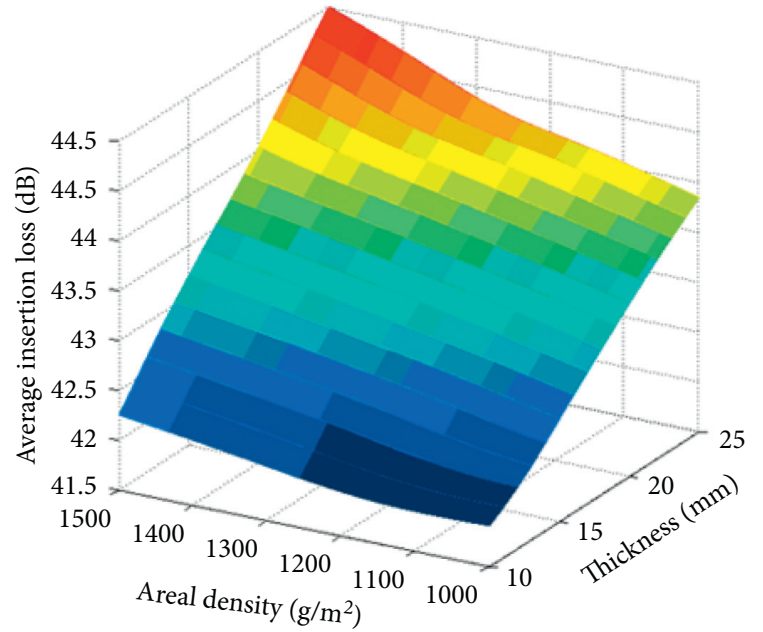

(a)

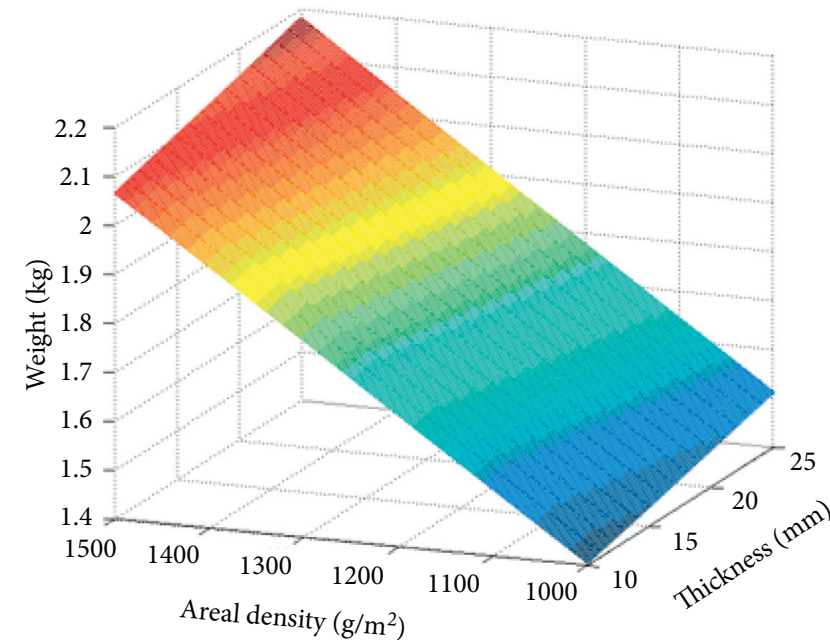

(b)

Figure 14: Approximate model of acoustic package for new material. (a) Average insertion loss, width, and surface density. (b) Weight, thickness, and surface density.

TABLE 9: Sample point used to test the accuracy of Kriging model.

\begin{tabular}{|c|c|c|c|c|c|}
\hline Test sample & Surface density of compress fiber $\left(\mathrm{g} / \mathrm{m}^{2}\right)$ & Thickness of glass fiber $(\mathrm{mm})$ & Average insertion loss $(\mathrm{dB})$ & Total weight $(\mathrm{kg})$ & $R^{2}$ \\
\hline 1 & 1091.261 & 21.560 & 43.604 & 1.608 & 0.95 \\
\hline 2 & 1338.912 & 23.359 & 44.224 & 1.950 & 0.90 \\
\hline 3 & 1458.605 & 19.504 & 43.879 & 2.081 & 0.97 \\
\hline 4 & 1408.768 & 13.426 & 42.825 & 1.970 & 0.92 \\
\hline 5 & 1018.073 & 14.860 & 42.538 & 1.462 & 0.97 \\
\hline 6 & 1206.668 & 12.434 & 42.388 & 1.695 & 0.99 \\
\hline 7 & 1132.591 & 16.420 & 42.957 & 1.625 & 0.94 \\
\hline 8 & 1356.686 & 11.481 & 42.391 & 1.887 & 0.93 \\
\hline 9 & 1277.997 & 18.187 & 43.425 & 1.831 & 0.93 \\
\hline 10 & 1150.496 & 24.603 & 44.079 & 1.709 & 0.98 \\
\hline
\end{tabular}




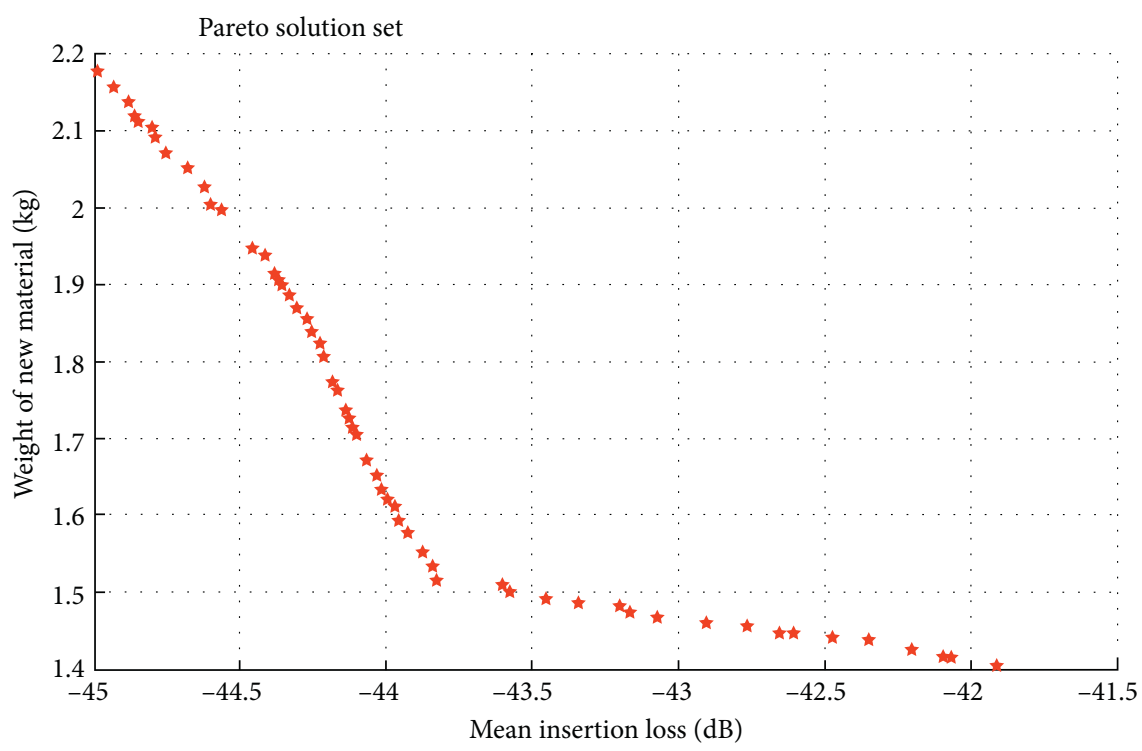

FIgURE 15: Pareto solution set between average insertion loss and total weight.

TABLE 10: Optimal values.

\begin{tabular}{lccc}
\hline Classes & Average insertion loss $(\mathrm{dB})$ & Total weight $(\mathrm{kg})$ & Computation time $(\mathrm{minutes})$ \\
\hline Result for SEA & 44.4734 & 1.9945 & 24.5 \\
Result for multiobjective genetic algorithm & 44.5629 & 1.9958 & 7.2 \\
Error \% & 0.2 & 0.065 & - \\
\hline
\end{tabular}

and proxy model method are used to study the sound insulation performance of front coamings, so as to obtain the influence of mass parameters, surface density parameters, and new material parameters on sound insulation performance. The results show that the sound insulation performance of the system is proportional to the increase of the coverage area and the coverage hole. The thickness of front coaming hole has great influence on sound insulation performance. Increasing the sound envelope thickness of front coamings has a more direct effect on the sound insulation performance at low frequency than at high frequency. The strict control of the leakage of the sound absorbing material and the hole greatly improves the sound insulation level.

(2) Multiobjective genetic algorithm and proxy model are used to obtain the optimal parameters of instrument panel sound package that meet the requirements of insertion loss and lightweight, and the acoustic performance is satisfied on the premise that the thickness does not increase.

(3) In this paper, the influence of acoustic material parameters and leakage cover parameters on acoustic packet insulation performance is obtained, which provides a new research direction for the research and development of acoustic packet.
(4) In the redesign method, the statistical energy model theory and the agent model theory can be used to carry out the forward design of acoustic packet system, which is an improvement on the existing acoustic packet design method.

\section{Data Availability}

The data used to support the findings of this study are included within the article.

\section{Conflicts of Interest}

The authors declare that they have no conflicts of interest.

\section{Acknowledgments}

This work was partially supported by the National Natural Science Foundation of China (Nos. 51275541 and 2017YFB0102603).

\section{References}

[1] J. H. Deng, "Effect of firewall assembly on vehicle soundpackage performance," Noise and Vibration Control, vol. 34, no. 3, pp. 78-82, 2014.

[2] X. H. Yu and J. D. Zhang, "Mechanism of inner noise generation of automobiles and the methods of control," Noise and Vibration Control, vol. 28, no. 5, pp. 122-125, 2008. 
[3] W. Xian, W. Cheng, S. Jianwang, and D. Guoming, "Dash sound package optimization based on Kriging model and NSGA-H algorithm," Vibration and Shock, vol. 35, pp. 231243, 2016.

[4] G. J. Pan, Analysis and improvement of passenger car's high frequency interior noise based on SEA method, Nanjing University of Aeronautics and Astronautics, Nanjing, China, 2014.

[5] F. Ge, Statistical energy analysis method in a certain vehicle on the noise reduction, Shang Hai Jiao Tong University, Shanghai, China, 2013.

[6] D. Y. Yao and Q. Z. Wang, Analysis principle and application of statistical energy, Beijing Institute of Technology, Beijing, China, 1995.

[7] X. Z. Li and Q. B. Huang, "Statistical energy analysis and optimization simulation of vehicle interior noise," Noise and Vibration Control, vol. 3, pp. 29-32, 2005.

[8] Z. Y. Hao and Z. Y. Ding, "Rapid optimization and design of Mag-Based composite dash panel with SEA method," Journal of Tianjing University Science and Technology, vol. 48, no. 1, pp. 33-38, 2015.

[9] Z. G. Lu, The prediction and optimization of the mid-frequency acoustic problems on the automobile thin-wall component based on the hybrid FE-SEA method, Zhejiang University, Zhejiang, China, 2011.

[10] J. Pang, NVH Control of Automotive Body, China Machine Press, Beijing, China, 2015.

[11] H. L. Zhang, G. Jin, and W. F. Cao, "Design of acoustic package for automobile," Automobile Parts, vol. 4, pp. 112132, 2014.

[12] X. T. Yang, Z. Q. Gu, Z. D. Yang, G.-P. Dong, and C. Xie, "Multi-target optimization of multilayer sound absorption material combinations in passenger compartment of a car," Vibration and Shock, vol. 32, no. 4, pp. 21-25, 2013.

[13] X. L. Liu, Y. W. Chen, X. R. Xing, and Y. G. Chen, Optimized latin hypercube sampling method and its application, pp. 3236, National University of Defense Technology, Guizhou, China, 2011.

[14] Q. Zhang, Analysis of structural reliability based on Kriging model, Dalian University of Technology, Dalian, China, 2005.

[15] Q. Zhang and X. S. Li, "Analysis of structural reliability based on Kriging model," Computational Mechanics, vol. 2, pp. 175-179, 2006.

[16] W. Y. Tang, Research and application of genetic algorithm in structural optimization, Dalian University of Technology, Dalian, China, 2002. 